\title{
On the potential of linked-basin tidal power plants: an operational and coastal modelling assessment
}

\author{
Athanasios Angeloudis ${ }^{\mathrm{a}, \mathrm{b}, *}$, Stephan C. Kramer ${ }^{\mathrm{b}}$, Noah Hawkins ${ }^{\mathrm{b}}$, Matthew D. \\ Piggott $^{\mathrm{b}}$ \\ ${ }^{a}$ School of Engineering, Institute for Infrastructure and the Environment, University of \\ Edinburgh, UK \\ ${ }^{b}$ Department of Earth Science $\&$ Engineering, Imperial College London, UK
}

\begin{abstract}
Single-basin tidal range power plants have the advantage of predictable energy outputs, but feature non-generation periods in every tidal cycle. Linkedbasin tidal power systems can reduce this variability and consistently generate power. However, as a concept the latter are under-studied with limited information on their performance relative to single-basin designs. In addressing this, we outline the basic principles of linked-basin power plant operation and report results from their numerical simulation. Tidal range energy operational models are applied to gauge their capabilities relative to conventional, single-basin tidal power plants. A coastal ocean model (Thetis) is then refined with linked-basin modelling capabilities. Simulations demonstrate that linked-basin systems can reduce non-generation periods at the expense of the extractable energy output relative to conventional tidal lagoons and barrages. As an example, a hypothetical case is considered for a site in the Severn Estuary, UK. The linked-basin system is seen to generate energy $80-100 \%$ of the time over a spring-neap cycle, but harnesses at best $\approx 30 \%$ of the energy of an equivalent-area single-basin design.
\end{abstract}

Keywords: Linked-basin lagoon, Tidal range energy, Resource assessment, Numerical model

\section{Introduction}

Tidal energy is a renewable energy source that comes with near complete predictability as a result of the tide generating forces attributed to the coupled Earth-Moon-Sun system. Coastal sites featuring amplified tidal velocities or ele5 vations can be sources of substantial marine energy [1, 2. As an example, a first array of tidal stream turbines has been installed in the Pentland Firth, Scotland,

\footnotetext{
* Corresponding author

Email address: a.angeloudis@ed.ac.uk (Athanasios Angeloudis )
}

Preprint submitted to Renewable Energy

March 10, 2020 
UK [3. The Pentland Firth is a prime example of a site that features amplified tidal currents that justify the deployment of marine hydrokinetic devices. Interest in tidal range structures has also resurfaced following the construction of the Lake Sihwa tidal power station in South Korea 4, while promising sites in the Bristol Channel and the Severn Estuary [5, 6] have been identified for their potential [7].

Tidal range power plants make use of hydraulic structures to facilitate elevation (pressure head) differences which drive flow through low-head hydro15 turbines and thus generate power. Theoretically, the maximum potential energy available from a tidal head difference $H(\mathrm{~m})$ is given in $\mathrm{J}$ by the classical study of Prandle [8] as:

$$
E_{\text {max }}=\frac{1}{2} \rho g A H^{2},
$$

where $\rho$ is the fluid density $\left(\mathrm{kg} / \mathrm{m}^{3}\right), g$ is the gravitational acceleration $\left(\mathrm{m} / \mathrm{s}^{2}\right)$ and $A$ is the impounded plan surface area $\left(\mathrm{m}^{2}\right)$.

20 The optimal strategy for harnessing the energy resource will depend upon site-specific constraints. As such, assessment of tidal range energy options prior to their development relies on models that simulate, predict and optimise their operation. Initially, reduced-order operation models that neglect hydrodynamic impacts (i.e. 0-D modelling) are appropriate for preliminary assessments and

25 optimisation analyses 9. Predictions from these simplified models must be treated with caution as hydrodynamics can be altered both in the far-field and in the immediate near-field [10. Such limitations together with advances in computational resources has driven the development of multi-dimensional (1-D, 2-D, 3-D) hydro-environmental models [11, 12] coupled with operation algo-

30 rithms of single-basin power plants [13, 14, 15, 16. In particular, these coupled modelling results highlight how 0-D models may overestimate the energy output by as much as $50 \%$ [17, 18, 19]. The level of overestimation depends on the way designs interfere with established tidal dynamics. Crucially, studies using tidal range energy models [20] show that single-basin tidal power plants are unable to

35 generate power over certain periods of time, in the absence of a sufficient head difference.

In contrast, linked-basin systems are fundamentally designed to deliver more consistent power contributions to the electricity grid. Whilst conventional tidal energy options are more predictable in comparison with other renewables [20,

40 linked-basin tidal energy concepts can in principle supplement this predictability by facilitating base load supply. The use of connected basins to control the energy captured from the tides is not new. Preliminary schematics date back to the $18^{\text {th }}$ century when Bernard Forest de Belidor presented designs to grind flour over a longer period of time than tidal mills by using multiple bodies of water [21, 22]. Descriptions of thirteen multi-basin concepts were summarised by Bernshtein 23 , drawing information from ideas since the $19^{\text {th }}$ century in an attempt to provide baseline power. Only a single small-scale scheme of this type has been recorded: the Haishan tidal power plant in China, featuring a capacity of $0.25 \mathrm{MW}$ since 1975 24, 25]. Linked-basin tidal power systems have been 
considered for other sites such as the Bay of Fundy [26] and as part of the Severn Estuary tidal power feasibility studies in the UK. However, Van Walsum [27, 26] reported that linked-basin proposals were typically dismissed in favour of singlebasin or paired/associated-basin configurations citing a lack of competitiveness in extracting energy at the lowest unit cost.

55 Linked-basin systems were studied conceptually, in the absence of hydrodynamic modelling [27, where assessment relied on analytical or 0-D modelling [23, 26]. As such, there is uncertainty over previous findings that do not acknowledge either hydrodynamics impacts or recent turbine developments that can be parameterised in the operational modelling.

We provide extensions to a methodology on the assessment of single-basin tidal range structures to examine a simple type of a linked-basin system. The overarching objective of the study is to provide an updated assessment on multibasin plants by optimising the operation of a representative design. In particular, to the best of our knowledge this is the first quantitative study on linkedbasin designs through standalone operational modelling that is then coupled within a coastal hydrodynamics model. Initially, the assessment is performed under idealised scenarios to deliver insight into how the technology functions and performs. We then consider a practical example, revealing technical considerations that would arise under realistic conditions.

\section{Methodology}

\subsection{Operation simulations of tidal power plants}

For tidal power plant operation simulations we employ: (a) a finite difference operational model based on the principles of mass-balance, and (b) the finite element-based coastal ocean model Thetis that solves the depth-averaged shal-

75 low water equations. Details of these tools in the context of single-basin tidal power plant assessment and optimisation are described in Angeloudis et al. [14]. Below we summarise the most salient details of the formulations applied for linked-basin systems.

\subsubsection{Conventional tidal range plant operation}

80

There is a wealth of information on the operation of single-basin power plants [20]. Their design entails the distribution of turbines and sluice gates along an impoundment. The impoundment encloses an area of water that is exchanged with the sea through the turbines for power generation (Fig. 1a,b). The plant operation can be either one-way (ebb-generation-only/flood-generation85 only, Fig. 2a), two-way (bidirectional generation, Fig. 2b) and even include pumping intervals for storage purposes. The duration of no-generation periods depends on tidal conditions (e.g. resulting from the significant variability over spring-neap cycles) and turbine capabilities [13. Fig. 2a-b illustrates the general sequence for ebb and two-way operation, highlighting periods of sufficient 90 head difference to drive the turbines (in grey). Effectively, the control of singlebasin tidal power plants depends solely on the difference between the inner $\left(\eta_{i}\right)$ 
(a)

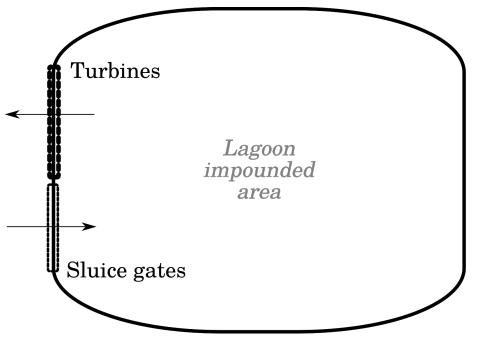

(b)

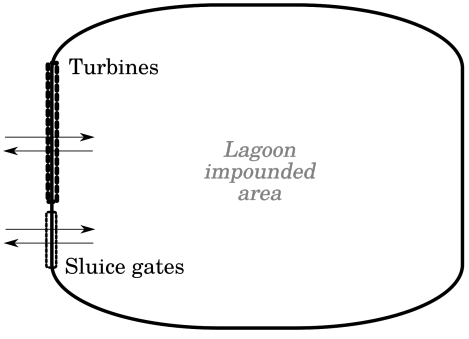

(c)

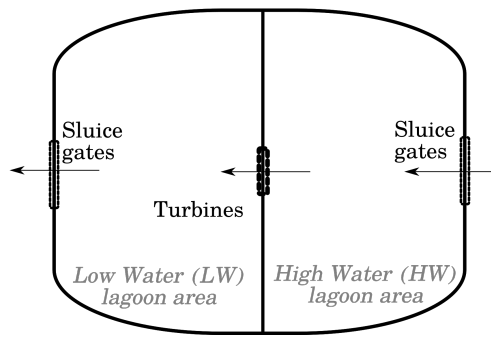

Figure 1: Tidal range power plant concepts: (a) single-basin ebb-only, (b) single-basin two-way and (c) linked-basin operation designs

and outer water levels $\left(\eta_{o}\right)$ in close proximity to the hydraulic structures. The head difference can be calculated as $H=\eta_{i}-\eta_{o}$ (see Fig. 2).

\subsubsection{Linked-basin system operation}

95 In their simplest form, linked-basin systems feature two lagoons/basins that are internally connected, as in Fig. 1k. The operation in time is illustrated in Fig. 22 demonstrating how a sufficient head difference $\left(H_{H W, L W}\right.$, Fig.2 $)$ can be theoretically maintained to generate power continuously. All turbines are distributed at an internal barrier splitting the impounded area into two

lagoons. The lagoons are separately connected to the sea through independent sluice gate sections. In contrast to single-basin designs, linked basin systems operate based on three head differences. Firstly, we have the head difference $\left(H_{H W, L W}\right)$ between the two connected basins that drives flow through turbines 

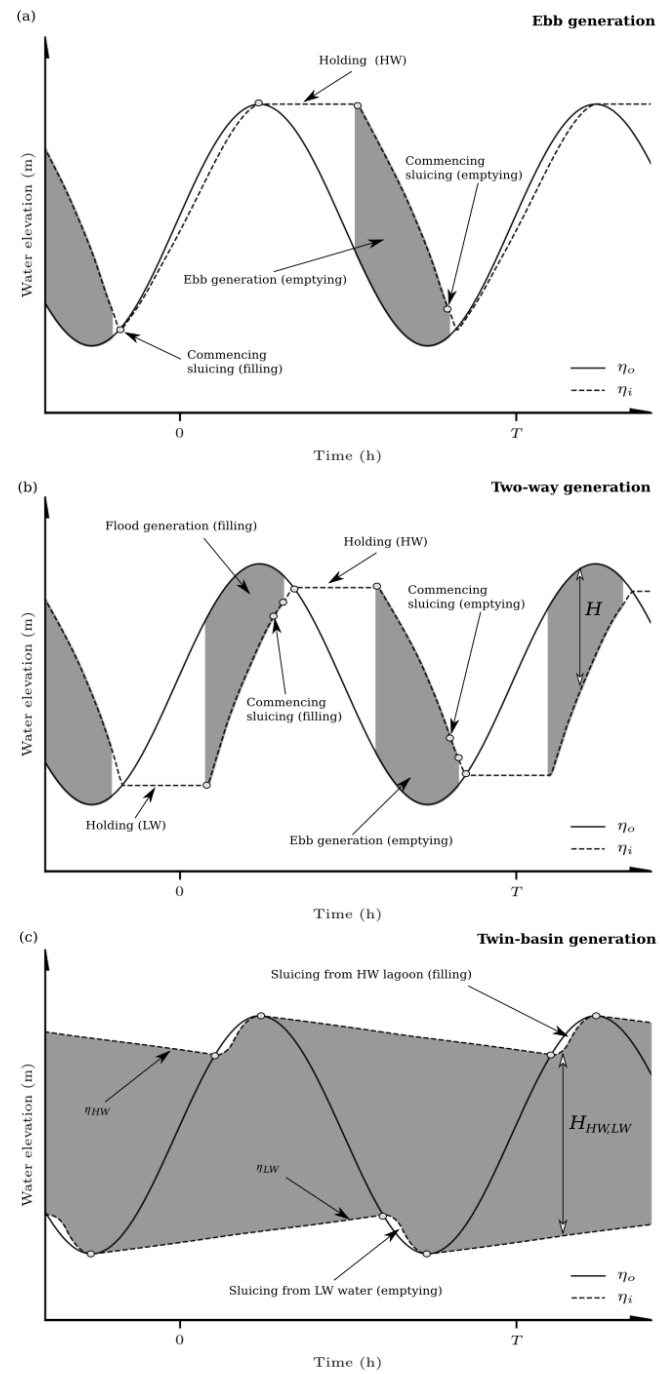

Figure 2: Operation of a tidal power plant over an $M_{2}$ tidal period, illustrating typical modes of operation. (a) Conventional one-way ebb generation, (b) two-way generation and (c) twinbasin generation. The dark grey sections indicate periods when $H$ drives power generation from the turbines. Single-basin control parameters used in the optimisation, such as the holding periods on ebb $\left(t_{h, e}\right)$ and flood $\left(t_{h, f}\right)$, are annotated for completeness.

at the internal barrier. We then have the two head differences at hydraulic structures connecting each basin to the sea. These latter head differences drive flow exchange between the linked-basin system and the sea. A practical example of a twin-basin system is presented in Fig. 3. Seawater enters a High Water $(H W)$ lagoon where sluice gates are regulated to maintain the highest water level 


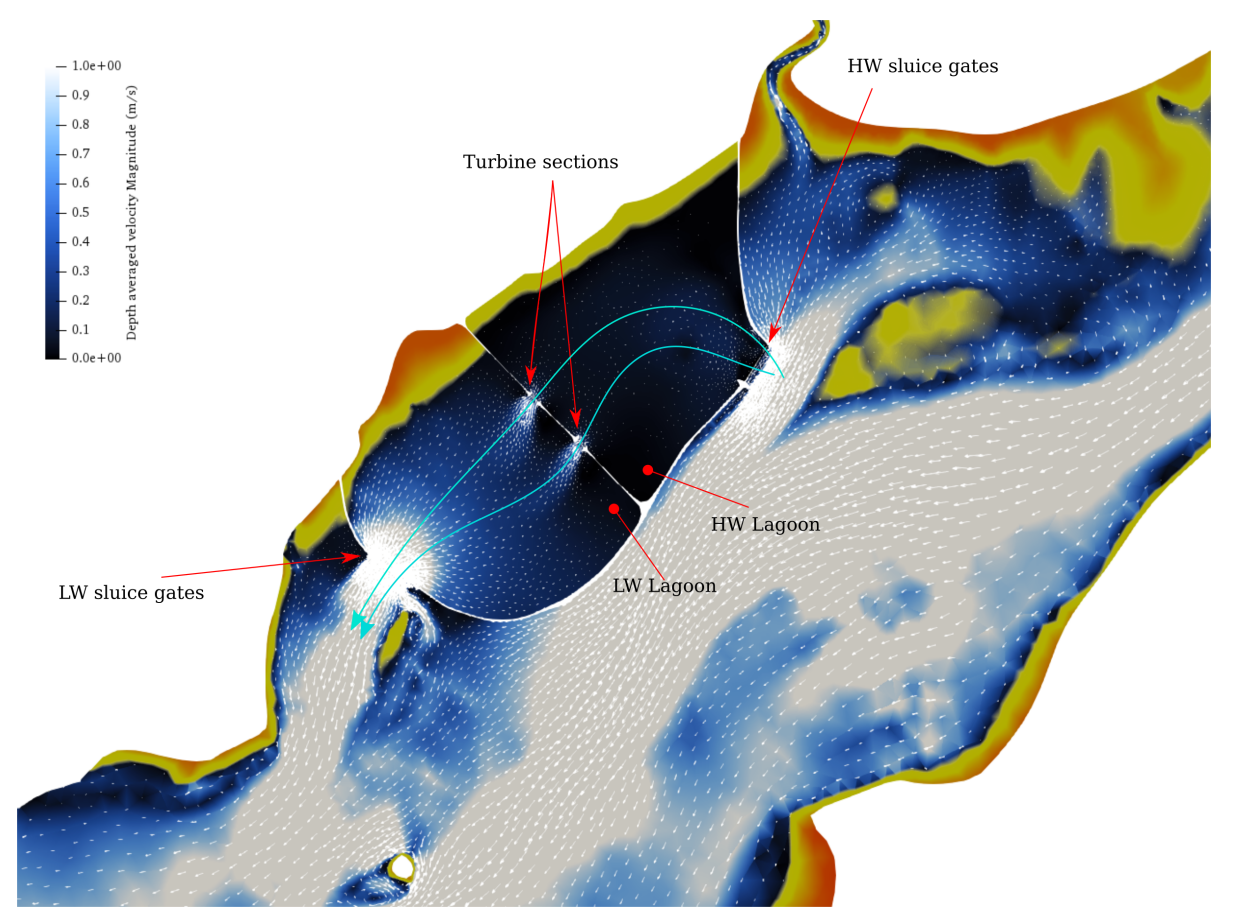

Figure 3: A practical example of a hypothetical twin-basin system that is discussed in detail later in this study. Shades of blue colour and vectors demonstrate instantaneous velocities from the operation of a hypothetical twin-basin system. The teal coloured trajectories illustrate indicative flow paths through the twin-basin system, passing first through the high (HW) and then the low water (LW) lagoons. Yellow/red regions indicate the dry areas within intertidal zones at the particular point in time.

possible. In the example of Fig. 3 this corresponds to the northern impounded area. The increased water elevation in the HW lagoon can be noted from the fact that the shoreline is positioned further inland relative to the other, Low Water $(L W)$ lagoon. The sluice gate discharge for the incoming flow in the HW lagoon $\left(Q_{s, H W}\right)$ is calculated using the orifice equation as

$$
Q_{s, H W}\left(H_{H W, o}\right)=\left\{\begin{array}{ll}
r(t) \cdot C_{d} \cdot A_{s, H W} \cdot \sqrt{2 g\left|H_{H W, o}\right|} & \text { for } H_{H W, o}<0.0 \\
0 & \text { for } H_{H W, o} \geq 0.0
\end{array},\right.
$$

where $A_{s, H W}$ is the aggregate cross-sectional flow area $\left(\mathrm{m}^{2}\right)$ of the gates in-

stalled, $C_{d}$ is the sluice gate discharge coefficient [28, and $r(t)$ is a ramp function introduced for numerical stability and a representation of the gradual opening/closing of hydraulic gates [29]. The value of $H_{H W, o}$ is the head difference between the HW lagoon inner water level $\left(\eta_{H W}\right)$ and the outer water level $\left(\eta_{o}\right)$ at the sluice gate location $\left(H_{H W, o}=\eta_{H W}-\eta_{o}\right)$. Similarly, there is a Low Water 
$(L W)$ lagoon where water is maintained at the lowest level possible (in Fig. 3 the LW lagoon is the southern impounded area). The LW lagoon sluice gate discharge, releasing water back into the sea $\left(Q_{s, L W}\right)$, is described by

$$
Q_{s, L W}\left(H_{L W, o}\right)=\left\{\begin{array}{ll}
-r(t) \cdot C_{d} \cdot A_{s, L W} \cdot \sqrt{2 g\left|H_{L W, o}\right|} & \text { for } H_{L W, o}>0.0 \\
0 & \text { for } H_{L W, o} \leq 0.0
\end{array},\right.
$$

where $A_{s, L W}$ is the aggregate cross-sectional flow area (in $\mathrm{m}^{2}$ ) of the LW lagoon sluice gates and $H_{L W, o}$ is the head difference between the LW lagoon inner water level $\left(\eta_{L W}\right)$ and $\eta_{o}$ at the sluice gate location. Sluice gates aim to maintain a sufficient head difference $\left(H_{H W, L W}\right)$ across turbine sections connecting the basins. The turbine flowrate $Q_{t}$ and power output $P$ are calculated as:

$$
\begin{gathered}
Q_{t}\left(H_{H W, L W}\right)= \begin{cases}r(t) \cdot N_{t} \cdot Q_{h}\left(H_{H W, L W}\right) & \text { for } H_{H W, L W} \geq h_{\min } \\
0 & \text { for } H_{H W, L W}<h_{\min }\end{cases} \\
P\left(H_{H W, L W}\right)= \begin{cases}r(t) \cdot N_{t} \cdot P_{h}\left(H_{H W, L W}\right) & \text { for } H_{H W, L W} \geq h_{\min } \\
0 & \text { for } H_{H W, L W}<h_{\min }\end{cases}
\end{gathered}
$$

where $N_{t}$ is the number of turbines. $Q_{h}$ and $P_{h}$ represent the turbine flow and power of individual turbines as per the parametrisation of Table 1. In

130 summary, taking Fig. 3 as an example, water through the twin-basin system follows a consistent pathway. It (a) enters by the HW sluice gates, (b) flows through the turbines connecting the HW and LW lagoons, generating energy, and (c) returns to the sea as the tide ebbs through the LW lagoon sluice gates.

\subsection{Tidal power plant design \& operation optimisation}

135 Single-basin power plants typically aim to maximise energy output rather than sustain a continuous power generation profile. In contrast, for linked-basin systems, consistent power generation becomes a priority. Differences in design objectives are highlighted by the optimisation functions defined in the following sections. The optimisation problems presented are solved either using brute 0 force computation or through the SciPy implementation of the Limited-memory Broyden-Fletcher-Goldfarb-Shanno algorithm with bounds (L-BFGS-B) 30. The optimisation builds on earlier studies for the operational optimisation of tidal power plants [14, 15, where the optimisation strategy is discussed further.

\subsubsection{Single-basin power plant energy maximisation}

The operation of hydraulic structures and the duration of the individual modes (Fig. 2 in single-basin power plants can be varied, and thus optimised. In order to pose a mathematical optimisation problem, we encode the duration of the operation modes (e.g. the holding and sluicing duration on ebb/flood 
Table 1: Parametrisation of a double-regulated bulb turbine applied in the tidal range energy resource assessments to calculate Power $P_{h}$ and Discharge $Q_{h}$. Adapted from [14].

\begin{tabular}{|c|c|c|}
\hline$\#$ & Formulations & Description \\
\hline 1 & $S_{p}=\frac{2 \cdot 60 \cdot f_{g}}{G_{p}}$ & $\begin{array}{l}\text { Turbine speed } S_{p}(\mathrm{rpm}) \text {, where } f_{g} \text { is the grid } \\
\text { frequency }(\mathrm{Hz}) \text { and } G_{p} \text { the generator pole num- } \\
\text { ber. }\end{array}$ \\
\hline 2 & $n_{11}=\frac{S_{p} \cdot D}{\sqrt{|H|}}$ & $\begin{array}{l}\text { Unit speed } n_{11}(\mathrm{rpm}) \text { where } D \text { is the diameter } \\
(\mathrm{m}) \text {. }\end{array}$ \\
\hline 3 & $Q_{11}= \begin{cases}0.017 n_{11}+0.49, & n_{11} \leq 255 \\
4.75, & n_{11}>255\end{cases}$ & $\begin{array}{l}\text { Unit discharge } Q_{11} \text { from empirical equations of } \\
9 \text {. }\end{array}$ \\
\hline 4 & $Q^{*}=Q_{11} D^{2} \sqrt{|H|}$ & $\begin{array}{l}\text { Discharge estimate } Q^{*}\left(\mathrm{~m}^{3} / \mathrm{s}\right) \text { through turbine } \\
\text { for } H(\mathrm{~m}) \text {. }\end{array}$ \\
\hline 5 & $P^{*}=\min \left(\rho g Q^{*}|H|, C_{p}\right)$ & $\begin{array}{l}\text { Power for } Q^{*} \text { subject to the turbine capacity } C_{p} \\
(\mathrm{MW}) \text {. }\end{array}$ \\
\hline 6 & $Q_{h}=\frac{P^{*}}{\rho g|H|}$ & $\begin{array}{l}\text { Correction of } Q^{*} \text { to determine } Q_{h} \text { in case of } \\
\text { maximum capacity } C_{p} .\end{array}$ \\
\hline 7 & $\eta_{h}=-0.0019 n_{11}+1.2461$ & $\begin{array}{l}\text { Empirical expression for hydraulic efficiency } \\
\eta_{h}[13 .\end{array}$ \\
\hline 8 & $\eta_{o}=\eta_{1} \cdot \eta_{2} \cdot \ldots \cdot \eta_{n-1} \cdot \eta_{n}$ & $\begin{array}{l}\text { Consideration of other efficiency factors, e.g. } \\
\text { turbine orientation, friction. }\end{array}$ \\
\hline 9 & $P_{h}=\rho g Q_{h}|H| \eta_{o}$ & $\begin{array}{l}\text { Power } P_{h}(\mathrm{MW}) \text { calculated subject to efficiency } \\
\text { losses. }\end{array}$ \\
\hline
\end{tabular}

tides) in a vector, $\boldsymbol{\tau}=\left\{t_{i}, i=1, \ldots, N\right\}$, where $N$ is the number of control variables over the simulation period spanning $t_{s}$. Accordingly, the following objective function is used to seek an optimal $\boldsymbol{\tau}$ and turbine number $\left(N_{t}\right)$ :

$$
\begin{array}{ll}
\max & f\left(\boldsymbol{\tau}, N_{t}\right)=I_{o}\left(\boldsymbol{\tau}, N_{t}\right)-C_{o}\left(N_{t}\right) \\
\text { subject to : } & \\
& \boldsymbol{\tau}_{l} \leq \boldsymbol{\tau} \leq \boldsymbol{\tau}_{u} \\
& 0.0 \leq N_{t} \times l_{t} \leq L
\end{array}
$$

where $L$ is the available impoundment length for the placement of hydraulic structures $(\mathrm{m}), l_{t}$ is the length occupied by individual turbines $(\mathrm{m})$. The vectors $\boldsymbol{\tau}_{l}$ and $\boldsymbol{\tau}_{u}$ refer to the lower and upper bounds expected for each mode of operation. For simplicity, we relate the sluice gate number $\left(N_{s}\right)$ with $N_{t}$ through the expression

$$
N_{s}=\frac{L-N_{t} \cdot l_{t}}{A_{s} / h_{s}},
$$

where $h_{s}$ is the cross-sectional height of the sluice gates installed. The ratio $h_{s} / A_{s}$ represents the sluice gate area per unit length. The prevailing assumption of Eq. 7 is that available hydraulic structure length not utilised by turbines is 160 occupied by sluice gates. The objective function of Eq. 6 is formulated as the difference of operation revenue $I_{o}$ (e.g. in $\left.£\right)$ and maintenance cost $C_{o}(£)$ over $t_{s}$. These quantities can respectively be calculated as

$$
I_{o}\left(\boldsymbol{\tau}, N_{t}\right)=\int_{t_{0}}^{t_{s}} V_{e} P\left(\boldsymbol{\tau}, N_{t}\right) d t,
$$




$$
C_{o}\left(N_{t}\right)=\int_{t_{0}}^{t_{s}} V_{o} N_{t} C_{p} d t,
$$

where $V_{e}$ and $V_{o}$ correspond to indicative values for energy (e.g. in $£ / \mathrm{MWh}$ ) and Operational and Maintenance (O\&M) costs per unit capacity installed (e.g. in $£ / M W$ ). The specific values selected can be informed by technical reports that estimate typical costs of tidal range energy, e.g. [31, 32]. The $C_{o}$ quantity of Eq. (9) is introduced to account for additional O\&M costs in replacing sluice gates with turbines. $C_{p}$ is the capacity of the installed turbines. For the 20 MW turbines considered, values of $V_{o}=2.5$ and $3.125 £ / \mathrm{MW} / \mathrm{h}$ are assumed for one-way and two-way configurations respectively. For two-way setups $V_{o}$ is greater to reflect the further maintenance required.

\subsubsection{Twin-basin power plant performance optimisation}

On twin-basin systems, we extend the optimisation function in Eq. 6 to include the standard deviation of the capacity factor $\left(C_{F}=P / C_{p}\right)$ over $t_{s}$.

175 This is imposed to establish a balance between energy maximisation and power generation profile consistency. The function is expressed as follows:

$\max$

$$
f\left(N_{t}\right)=\frac{1}{1+\beta \sigma_{P}} I_{o}\left(N_{t}\right)-C_{o}\left(N_{t}\right)
$$

subject to :

$$
0.0 \leq N_{t} \times l_{t} \leq L
$$

where $\sigma_{P}$ is the standard deviation of power generated and $\beta$ a weighting factor amplifying the significance of continuous power output at the expense of the overall energy produced. Contrary to the function defined in Eq. (6), the vector $\boldsymbol{\tau}$ is no longer applicable, with $N_{t}$ remaining the only control parameter. In achieving a consistent power output, rather than controlling the flow through turbines to deliver a uniform amount of energy, the algorithm aims to maintain a head difference $H_{H W, L W}$ that is greater than the turbine rated head difference $\left(H_{r}\right)$ [13. This allows installed turbines to generate power at their maximum 185 generation capacity. The hill chart parametrisations (see Table 1) assume that turbines always operate at their peak possible efficiency $\left(\eta_{h}\right)$ and do not account for potential tuning capabilities. This assumption is made in the absence of commercially sensitive details from specific turbine manufacturers.

\subsection{Hydrodynamic Modelling}

190 Thetis (http://thetisproject.org/) is a 2-D/3-D model for coastal and estuarine flows. It is implemented using the Firedrake finite element Partial Differential Equation (PDE) solver framework [33, 34. We consider the nonconservative form of the nonlinear shallow water equations:

$$
\frac{\partial \eta}{\partial t}+\nabla \cdot\left(H_{d} \mathbf{u}\right)=0,
$$




$$
\frac{\partial \mathbf{u}}{\partial t}+\mathbf{u} \cdot \nabla \mathbf{u}-\nu \nabla^{2} \mathbf{u}+f \mathbf{u}^{\perp}+g \nabla \eta=-\frac{\tau_{b}}{\rho H_{d}},
$$

where $\eta$ is the water elevation, $H_{d}$ is the total water depth, $\mathbf{u}$ is the depthaveraged velocity vector, and $\nu$ is the kinematic viscosity of the fluid. The term $f \mathbf{u}^{\perp}$ represents the Coriolis "force", where $\mathbf{u}^{\perp}$ is the velocity vector rotated counter-clockwise over $90^{\circ}$, and $f=2 \Omega \sin (\zeta)$ with $\Omega$ the angular frequency of the Earth's rotation and $\zeta$ the latitude. Bed shear stress $\left(\tau_{b}\right)$ effects are represented here through Manning's $n$ formulation as:

$$
\frac{\tau_{b}}{\rho}=g n^{2} \frac{|\mathbf{u}| \mathbf{u}}{H_{d}^{\frac{1}{3}}} .
$$

Intertidal processes are treated using the wetting and drying formulation of Karna et al. 35]. The model is configured to use a discontinuous Galerkin finite element spatial discretisation (DG-FEM) and semi-implicit Crank-Nicolson timestepping for temporal discretisation. The nonlinear discretised shallow water equations are iteratively solved by Newton's method using the PETSc library [36. The representation of turbines and sluice gates is implemented through the domain decomposition approach outlined in Angeloudis et al. 37. Essentially, hydraulic structure fluxes linking subdomains are determined by firstly sampling water elevations. The elevation data is used to calculate $H$ values that feed into the hydraulic structure parametrisations (i.e. Eq. 25 and Table 1).

\subsection{Simulation setups for the assessment of linked-basin systems}

The assessment of the linked-basin power plant concept is conducted in two stages through comparisons against conventional single-basin power plants to emphasize differences on operation and performance. Firstly, idealised 0-D scenarios examine the sensitivity of tidal power plant performance to the tidal range 215 resource and the hydraulic structure configuration. Sequentially, operational (0D) and hydrodynamic (2-D) models compare a single-basin tidal lagoon design against a hypothetical linked-basin system in the Severn Estuary, UK.

\subsubsection{Idealised tidal range structures}

We consider an idealised setup for a tidal range structure that, has a fixed surface area $A$ and is not susceptible to intertidal effects. Seaward (outer) elevations $\left(\eta_{o}\right)$ are represented through a sinusoidal signal of period $T=12.42$ h (i.e. an $M_{2}$ tidal period) and amplitude $\alpha$. Four configurations are tested:

1. an ebb-only operation single-basin power plant (Fig. 2a) imposing an ebb holding period of $t_{h, e}=4.0 \mathrm{~h}$.

2. a two-way operation single-basin power plant (Fig. 2b) imposing ebb/flood holding periods of $t_{h, e}=t_{h, f}=3.0 \mathrm{~h}$.

3. a linked-basin system that is unconstrained in its operation relative to the variance of power output, i.e. $\beta=0$. 
4. a linked-basin system that is constrained to minimise the variance of power output by setting $\beta=25$ (see Eq. 10 ).

For simplicity, the operational control parameters $\left(t_{h, e}, t_{h, f}\right)$ for single-basin plants is explicitly specified using representative values 14 to limit the optimisation variables in Eq. 6 to the turbine number $N_{t}$ alone. Due to the singlevariable nature of the optimisation problem, $N_{t}$ is determined through brute force while exploring the full parameter-space. Sensitivity to the following is examined:

- The effect of tidal amplitude $(\alpha)$ on extractable energy over an annual period. In principle, the potential energy available is determined from Eq. 11, with empirical efficiency estimates of $27 \%$ and $37 \%$ for ebb and two-way respectively, as per Burrows et al. [19]. However, more recent optimisation studies suggest that these percentages can vary [14.

- The significance of the impoundment length $L$ available to position hydraulic structures. The sensitivity is based on a relationship $L=\lambda \times A$ where $\lambda(1 / \mathrm{m})$ is iteratively altered for a given impounded area $A$. While the resource for $A$ can be quantified, the actual energy harnessed will invariably be constrained by limitations on the siting of turbines and sluice gates (e.g. bathymetry, geomorphology and construction logistics) 38].

- The influence of $\beta$ in the twin-basin optimisation function of Eq. (10). This is meant to assess for linked-basin systems the compromise on the extractable energy if a more consistent generation profile is prioritised.

\subsubsection{A hypothetical case study within the Severn Estuary, UK}

Water elevations $(\eta)$ at any site of practical relevance are characterised by a variability from the interaction of multiple tide-generating forces caused by the relative positions of the Earth, Moon and Sun. These forces can be represented via a mathematical expansion of harmonic tidal constituents, as described in more detail by Parker [39]. Differences in the period of the tide-generating forces lead to the longer spring-neap (and other) cycles and thus the varying tidal range. In addition, other hydrodynamic, wind and wave events 12 will also play a role on the tidal elevations experienced by a power plant.

As an example, we start from a proposal by Tidal Lagoon Power Ltd (TLP) for a Cardiff Lagoon; this would be a tidal range structure occupying an area of $\approx 66 \mathrm{~km}^{2}$ within the Severn Estuary, UK. The general arrangement of the single-basin design studied previously [17, 40] is indicated in Fig. 4(c). We then consider a linked-basin system in the same location, once modifying the 265 single-basin configuration to ensure hydraulic structures are positioned in areas of sufficient depth (see Fig. 44) 38. Impoundment outline modifications are imperative as the siting of hydraulic structure sections would realistically take advantage of bathymetric features that minimise dredging during construction and maintenance. Eventually, the two lagoons making up the linked-basin system split the site into two approximately equal surface area basins of $33 \mathrm{~km}^{2}$ 
each. The configurations for both single-basin and linked-basin designs occupy the same area $\left(\approx 66 \mathrm{~km}^{2}\right)$ to ensure the two designs remain comparable, despite their unique design features.

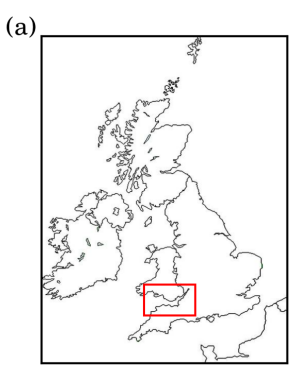

(d)

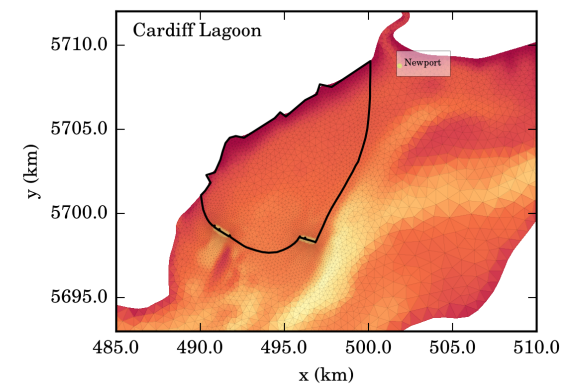

(b)

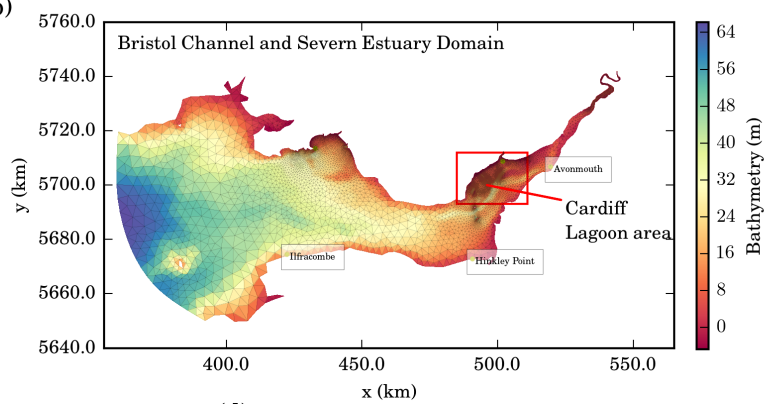

(d)

Figure 4: (a) Study area relative to UK map, (b) Computational domain considered for the simulations in the Bristol Channel and the Severn Estuary, (c) Cardiff Lagoon and (d) Cardiff twin-basin system and unstructured mesh refinement.

Unstructured meshes for the hydrodynamics simulations are generated using qmesh 41 (Fig. 4). The baseline simulation's unstructured mesh comprised 20,666 nodes and 41,342 elements and was setup to simulate the tidal hydrodynamics over a lunar month beginning on the $6^{\text {th }}$ of May 2003. This was preceded by a 5 -day simulation period that allowed the model to spin-up and become independent of the initial equilibrium conditions. Hydrodynamic models were forced at the seaward and inland boundaries using eight constituents from the TPXO database [42] and mean river flows from the UK's National River Flow Archive respectively. For the baseline scenario satisfactory agreement was demonstrated for the amplitudes and phases of the major constituents (Fig. 5); more details on the validation of Thetis models for the particular region are expanded in Angeloudis et al. 14]. Hydrodynamic modelling of the tidal range structure operation (Fig. 4k,d) entails the refinement of the baseline setup in the proximity of hydraulic structures. The domain is then split into subdomains that are connected through coupled open flux boundaries as 

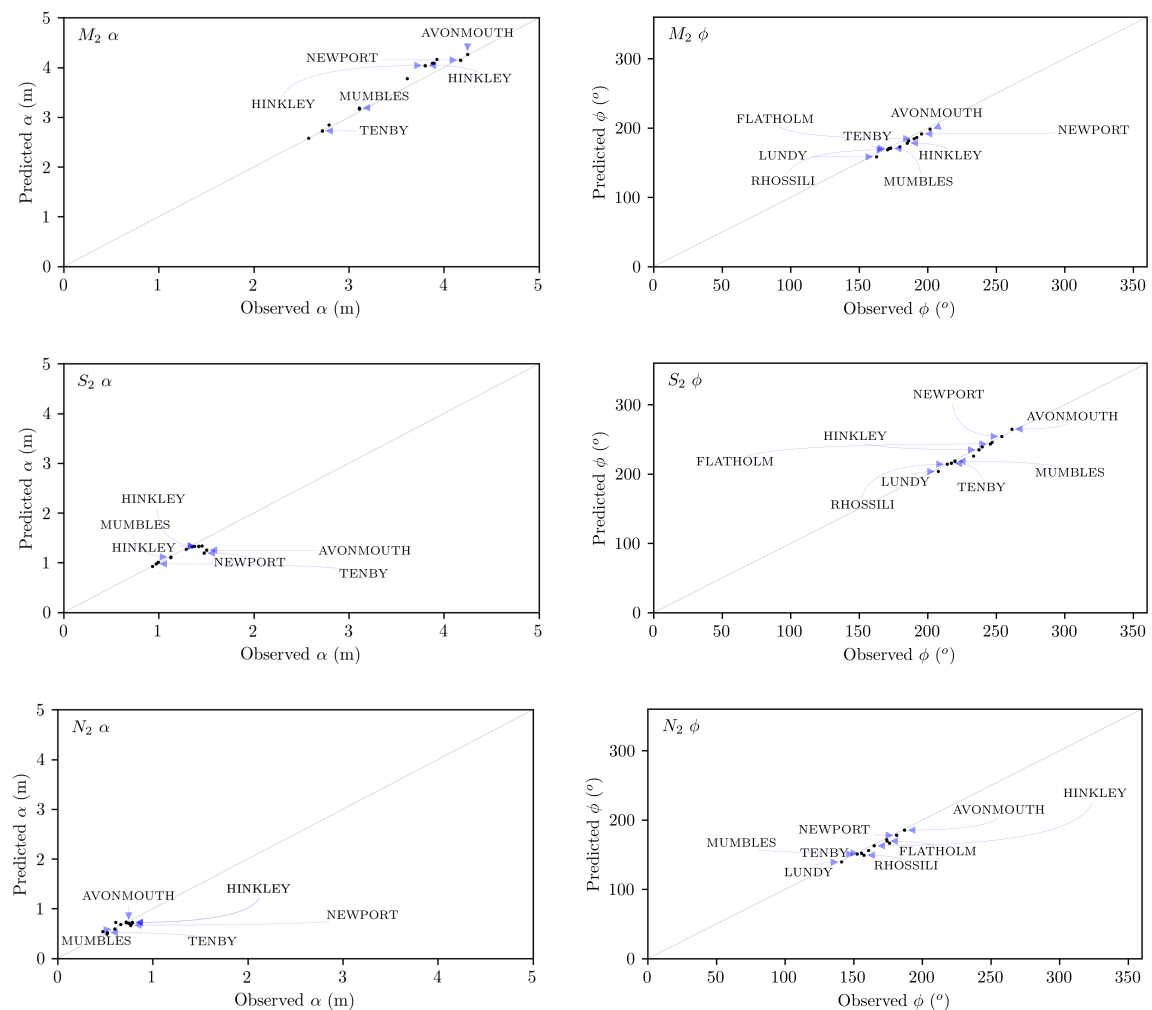

Figure 5: Comparison between predicted and observed tidal amplitude data for the three major tidal constituents in the region $\left(M_{2}, S_{2}\right.$ and $\left.N_{2}\right)$ for the baseline Bristol Channel and Severn Estuary model.

described previously 37. Different meshes are generated for the simulation of

e-basin and linked-basin systems by refining the baseline model. Despite their outline differences, identical mesh gradation criteria are imposed. Meshes are consistently refined to a resolution of $40 \mathrm{~m}$ at the hydraulic structure sections and $100 \mathrm{~m}$ in the proximity of the embankment separating the inner and outer water volumes. For the single-basin case, the baseline domain was split into a seaward domain covering the Bristol Channel and the Severn Estuary, and an inner one for the impounded area. Linked-basin simulations thus comprised three subdomains; one modelling the outer estuarine flow, and two for the basins forming the system (Fig. 4 4 ).

Water elevation time series predicted at the hydraulic structure locations are harmonically analysed to reconstruct extended time-elevation signals. The signals are used as inputs for the 0-D model as a representation of the seaward elevations at sluice gate and turbine sections. In addition, bathymetric data from the Edina Digimap Service 43 is employed to produce the plan surface area vs head difference relationships to parametrise the change in area due to 

surface area [8].

The turbine and sluice gate configuration for the single-basin design is initially determined by optimising Eq. 6 for both ebb-only and two-way generation cases. Optimisation is constrained by a hydraulic structure length of $L=2200 \mathrm{~m}$ 310 (corresponding to a value of $\lambda \approx 3 \times 10^{-5} \mathrm{~m}^{-1}$ in the idealised sensitivity study described in Section 2.4.1, chosen to be consistent with the outline proposal of TLP [40]. The optimisation function requires $0-\mathrm{D}$ simulations spanning a year from the $6^{\text {th }}$ of May 2003. An equivalent approach is then applied for the twin-basin system using the function in Eq. 10 for $\beta=0.0$.

Finally, the optimised setups are applied to examine:

- differences in the generation profile resulting from the operation strategy selected in single-basin designs;

- implications of aiming to generate continuously using a fixed number of turbines in linked-basin systems;

- impact of adjusting the number of turbines operating in order to sustain a continuous generation profile over certain periods;

- variations in power generation output and deviations among 0-D and 2-D modelling results.

\section{Results}

\subsection{Model predictions: Idealised tidal lagoons}

We first consider sensitivity studies in idealised single- and linked-basin systems. Results suggest that linked-basin systems produce substantially less energy than single-basin designs for the same surface area $(A)$ (Fig. 6). As long as there is sufficient space for the distribution of turbines (controlled here by the parameter $\lambda$ ), the plant efficiency for single-basin systems improves with an increasing tidal amplitude $(\alpha)$. This is expected given the superior generation efficiency of bulb turbines for a greater head difference $(H)$ [13]. For single-basin designs, as $\alpha$ increases, the optimisation converges towards using the allocated hydraulic structure area exclusively for turbines. Specifically, in Fig. 6f only 335 turbines (i.e. zero sluice gates) are installed once $N_{t} / \mathrm{km}^{2}$ transitions to a flat line. In contrast, linked-basin systems always require sluice gates at the seaward sides since these are crucial to the power generated from the internal barrier (see Fig 6e).

For values of $\lambda=6 \times 10^{-5}$ and $\alpha>2 \mathrm{~m}$, an idealised two-way configuration generates $\approx 30-50 \%$ of the theoretically available potential energy of Eq. 1 . The ebb-only design follows with an equivalent $\approx 20-37 \%$. A twin-basin scenario unconstrained to generate continuously $(\beta=0.0)$ only captures $\approx 15 \%$ of the resource. For a more consistent power output (i.e. $\beta=25.0$ ) the efficiency reduces to $\approx 3-14 \%$ (Fig. $6 \mathrm{p}$ ). 

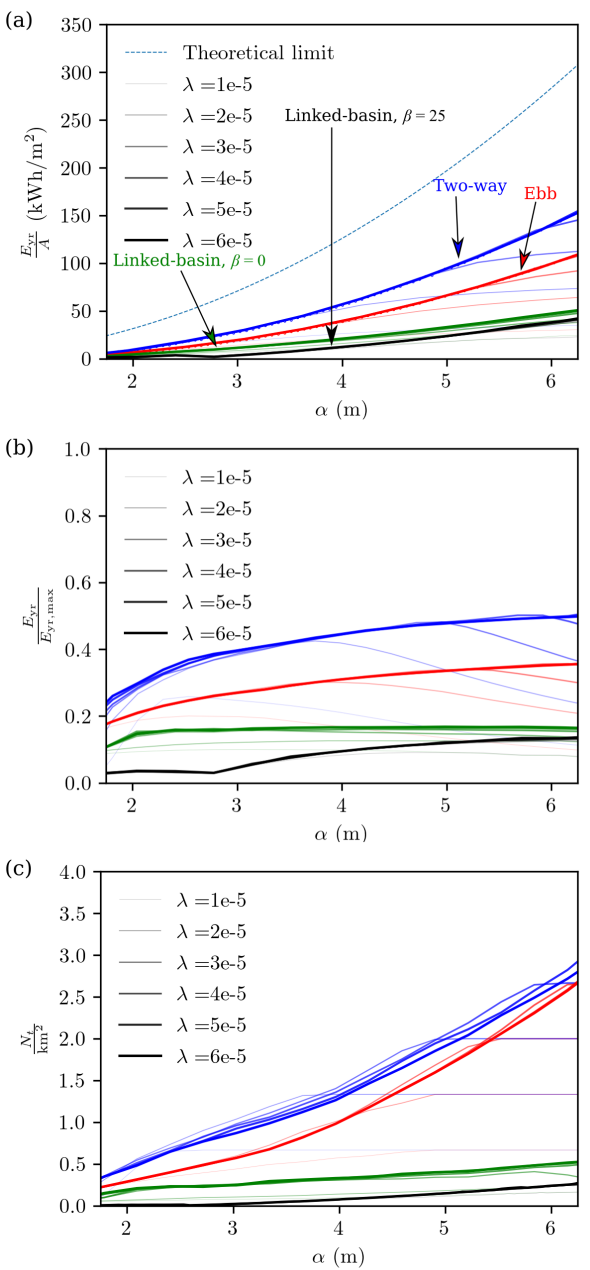

Figure 6: Sensitivity of tidal plant performance and configuration to amplitude $(\alpha)$. (a) Spatially normalised annual energy $\frac{E_{\mathrm{yr}}}{A}$, (b) generation efficiency relative to maximum potential energy $\frac{E_{\mathrm{yr}}}{E_{\mathrm{yr}, \max }}$, (c) normalised turbine number per area for idealised ebb (—), two-way

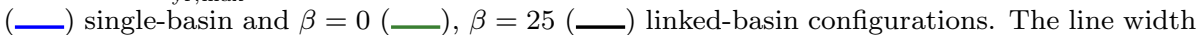
varies with the value of $\lambda$ that is applied to constrain the introduction of hydraulic structures to a finite length of the impoundment.

Even though twin-basin systems extract a lower fraction of the available energy, they require far less installed capacity (Fig. 6c), and potentially a lower O\&M cost, despite requiring more sluice gates to support $H_{H W, L W}$. Even though this sensitivity assessment (Fig. 6) sheds light on some generic characteristics of the performance of configurations described in Fig. 1] it excludes factors that can only be appreciated through a practical example. 


\subsection{Model predictions: Case study in the Severn Estuary, UK}

Analysis of our case study can be split into discrete sections. Given the baseline hydrodynamic model results, we first define the tidal power plant configuration through operational optimisation over an annual period. Once the design configurations have converged in terms of turbines and sluice gates, setups are prepared and run to assess the operation. Finally, 0-D modelling results are compared against the more computationally demanding Thetis results to reflect on the hydrodynamics impact.

\subsubsection{Tidal power plant configurations}

Single-basin optimisation results converge to a capacity of $1960 \mathrm{MW}$, which is within the range of $1800-2800 \mathrm{MW}$ proposed [40. The capacity corresponds to $98 \times 20 \mathrm{MW}$ turbines of diameter $D=7.35 \mathrm{~m}$ and $48 \times 150 \mathrm{~m}^{2}$ sluice gates (from Eq. 7). For a scenario assuming control parameters remain fixed over the simulation period, the plant performs best in ebb-only generation by imposing 365 an average holding time of $t_{h, e}=4.10 \mathrm{~h}$, whereas for two-way generation $t_{h, e}=$ $3.41 \mathrm{~h}$ and $t_{h, f}=2.30 \mathrm{~h}$ respectively.

In linked-basin systems, the optimal configuration maximising energy output is $24 \times 20 \mathrm{MW}$ turbines. This corresponds to a capacity $C_{p}=480 \mathrm{MW}$ and 110 sluice gates as per Eq. 7. Beyond this point, turbine and sluice gate configurations are assumed fixed for the cases summarised in Table 2

Cases presented highlight characteristics of the two types of tidal range structures. There is a set (i.e. SB-EBB-C, SB-TW-C, LB- $N_{t}=4$, LB- $N_{t}=10$, LB$\left.N_{t}=24\right)$ where control parameters are not altered as the tide evolves. Instead the control remains "Fixed", i.e. for single-basin plants the scheduling param375 eters remain the same, while for twin-basin systems the number of operational turbines $N_{t}$ does. The "Adaptive" operation strategies (i.e. the remaining cases listed in Table 2) aim to acknowledge the temporal variability of the tides. This is approached by solving the optimisation problems of Eqs. 6 and 10 for every tidal cycle to determine control parameters as the tide evolves. For single-basin 380 scenarios, holding periods $\left(t_{h, e}, t_{h, f}\right)$ are recursively updated in every tidal period. In linked-basin system scenarios, the number of operational turbines $N_{t}$ is regulated to promote consistent power outputs subject to $\beta$. 
Table 2: Cases considered for the assessment of twin-basin performance. $t_{h, e}=$ ebb holding period (h), $t_{h, f}=$ flood holding period, $N_{t, \max }=$ maximum turbine number $N_{t}$ that can be used.

\begin{tabular}{ll|lll}
\hline$\#$ & ID & Type & Details & Operation Description \\
\hline 1 & SB-EBB-C & Single-basin & $t_{h, e}=4.10 \mathrm{~h}, t_{h, f}=0.00 \mathrm{~h}$ & Fixed control ebb-only \\
2 & SB-TW-C & Single-basin & $t_{h, e}=3.41 \mathrm{~h}, t_{h, f}=2.30 \mathrm{~h}$ & Fixed control two-way \\
3 & SB-EBB-A & Single-basin & Variable $t_{h, e}$ & Adaptive $\boldsymbol{\tau}$ for ebb-only \\
4 & SB-TW-A & Single-basin & Variable $t_{h, e}, t_{h, f}$ & Adaptive $\boldsymbol{\tau}$ for two-way \\
5 & LB- $N_{t}=4$ & Linked-basin & $N_{t}=4$ & Fixed $N_{t}$ throughout \\
6 & LB- $N_{t}=10$ & Linked-basin & $N_{t}=10$ & Fixed $N_{t}$ throughout \\
7 & LB- $N_{t}=24$ & Linked-basin & $N_{t}=24$ & Fixed $N_{t}$ throughout \\
8 & LB- $\beta=5.0$ & Linked-basin & $N_{t, \max }=24, \beta=5.0$ & Adaptive $N_{t}$ based on $\beta$ \\
9 & LB- $\beta=10.0$ & Linked-basin & $N_{t, \max }=24, \beta=10.0$ & Adaptive $N_{t}$ based on $\beta$ \\
10 & LB- $\beta=25.0$ & Linked-basin & $N_{t, \max }=24, \beta=25.0$ & Adaptive $N_{t}$ based on $\beta$ \\
\hline
\end{tabular}

\subsubsection{0-D modelling results}

An overview of $0-\mathrm{D}$ modelling results is summarised in Table 3 Firstly, predictions of normalised energy outputs for single-basin plants using fixedcontrol configurations (SB-EBB-C, SB-TW-C) are aligned with previous studies. For example, the Burrows et al. [19] estimates of $27 \%$ and $37 \%$ for ebb-only and two-way generation are close to our predictions of $29.8 \%$ and $36.9 \%$ respectively. Operational optimisation in the adaptive setups delivers improved performance which is merely marginal $(\leq 1 \%)$ for ebb-only generation (SB-EBB-A) as only a single scheduling parameter is allowed to vary (ebb holding period, $t_{h, e}$ ) with minor impact on the output. However, the performance improvement for twoway generation $(\mathrm{SB}-\mathrm{TW}-\mathrm{A})$ following optimisation is more substantial $(\approx 5 \%)$. Ebb-only configurations generate $\approx 26.5 \%$ of the time whereas two-way setups generate for $\approx 43.5 \%$ of the time. There are discernible patterns in the generation profile of configurations using adaptive controls, with holding periods $\left(t_{h, e}, t_{h, f}\right)$ during neap tides being prolonged to maximise $H$ prior to turbining, leading to shorter power generation intervals. Over spring tides as greater $H$ values can be sustained, holding periods are shortened, leading to longer generation periods. 400 These trends can be appreciated in Fig. 7 a.

The linked-basin system output performance is notably worse relative to single-basin cases. The best scenario in terms of energy output $\left(\mathrm{LB}-N_{t}=24\right)$ extracts $42.5 \%$ and $29.7 \%$ of the optimised ebb (SB-EBB-A) and two-way (SBTW-A) configurations respectively. However, the particular linked-basin system $\left(\right.$ LB- $\left.N_{t}=24\right)$ has an availability of only $80 \%$ as the use of $N_{t}=24$ turbines leads to a rapid depletion of $H_{H W, L W}$ resulting in frequent no-generation periods (Fig. $7 \mathrm{~b}$, blue lines). Reducing the number of turbines (LB- $\left.N_{t}=10\right)$ allows consistent power generation during spring tides, but not over neap tides. The reduced tidal range during neap tides does not permit the condition of $H_{H W, L W}>H_{r}$

410 to be sustained indefinitely (Fig. 7b, green lines).

The concept of regulating the number of operational turbines in linked-basin systems returns interesting results. The power generation profiles in Fig. 7 ; suggest that as $\beta$ is increased, the number of operational turbines at neap tides is reduced to limit the water volume exchange between HW and LW lagoons 

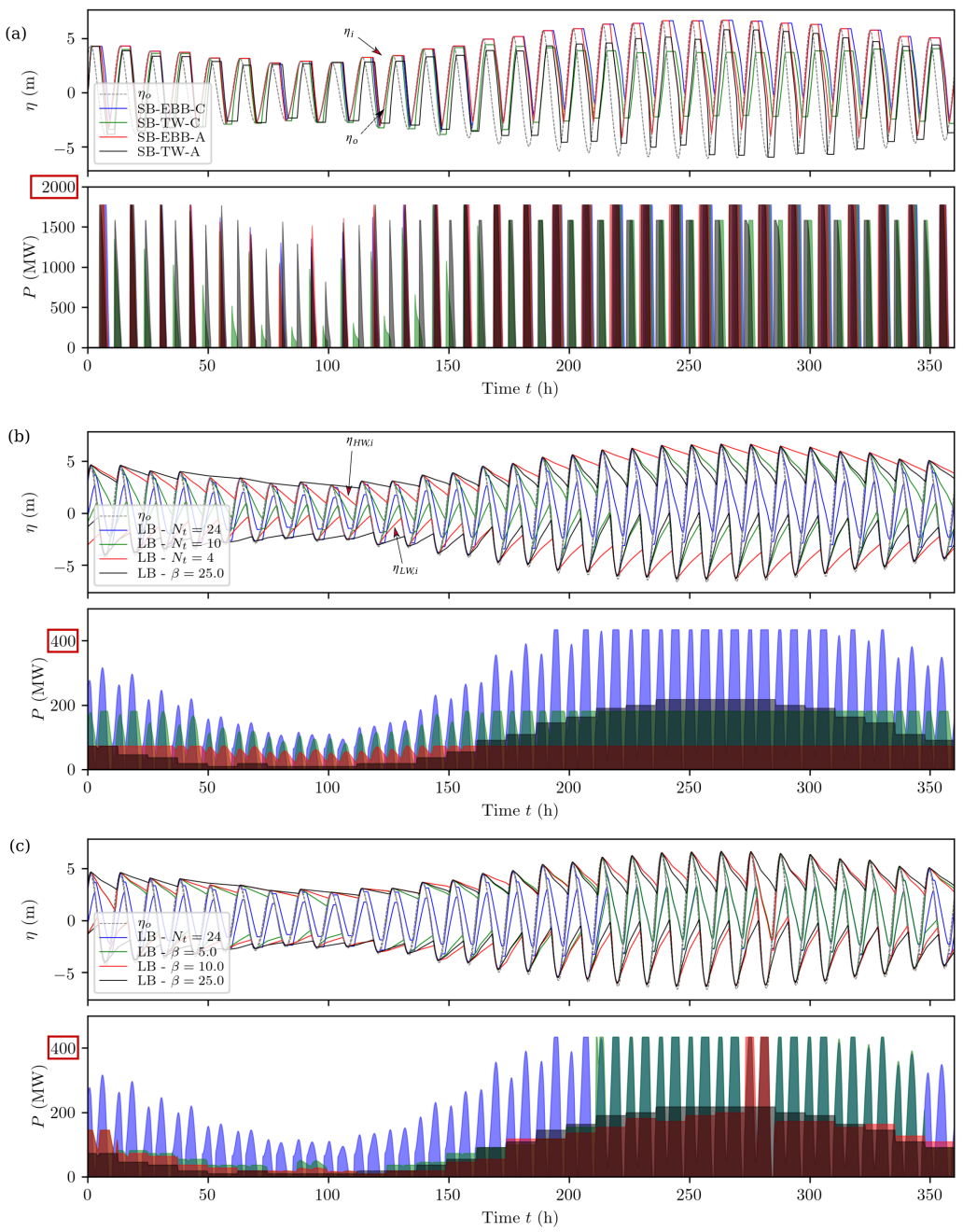

Figure 7: Elevations and power output of single- and linked-basin cases over a spring-neap cycle as predicted by OD modelling. (a) Single-basin cases; (b) Comparison of linked-basin cases using a fixed number of turbines - including the adaptive operation linked-basin case with $\beta=25.0$ (LB- $\beta=25.0$ ); (c) Comparison of linked-basin cases adapting the number of operational turbines temporally - including a case where the number of operational turbines remains fixed (LB- $\left.N_{t}=24\right)$. The range of power $P$ for in each plot is highlighted to emphasize differences in magnitude of power generated among single- and linked-basin designs.

to preserve $H_{H W, L W}$ and deliver a constant power output over every $12.42 \mathrm{~h}$ period; this corresponds to substantially reduced total power generation. With a transition to spring tide the number of operational turbines increases, enhancing power generation. For low values of $\beta$ (i.e in this case $\beta=5$ and 10) even though the function in Eq. 10 penalises output based on its consistency, the 
Table 3: 0-D operational model power output predictions. $E_{y r, 0 D}=0$-D predicted annual energy output, $E_{\max }=$ maximum theoretical energy available $[1],\left|C_{F}\right|=$ average capacity factor throughout the year. Availability is defined as the proportion of time with non-zero generation.

\begin{tabular}{l|cccc}
\hline Configuration & $E_{y r, 0 D}(\mathrm{TWh})$ & $E_{y r, 0 D} / E_{\max }(\%)$ & $\left|C_{F}\right|(\%)$ & Availability (\%) \\
\hline SB-EBB-C & 3.131 & 29.8 & 18.2 & 26.4 \\
SB-EBB-A & 3.180 & 30.3 & 18.5 & 26.7 \\
SB-TW-C & 3.871 & 36.9 & 22.6 & 42.8 \\
SB-TW-A & 4.558 & 43.4 & 26.6 & 44.1 \\
LB- $N_{t}=4$ & 0.566 & 5.4 & 80.7 & 100.0 \\
LB- $N_{t}=10$ & 1.061 & 10.1 & 60.6 & 97.6 \\
LB- $N_{t}=24$ & 1.354 & 12.9 & 30.9 & 80.1 \\
LB- $\beta=5.0$ & 1.040 & 9.9 & 23.7 & 97.4 \\
LB- $\beta=10.0$ & 0.732 & 6.9 & 16.7 & 99.9 \\
LB- $\beta=25.0$ & 0.630 & 6.0 & 27.7 & 100.0 \\
\hline
\end{tabular}

algorithm converges to using all available turbines (i.e. $N_{t}=24$ ). For $\beta=25$, the power output remains flat for all tidal cycles, whilst regulating the number of operational turbines according to gradual changes in the tidal range.

Insights into the generation profiles of single-basin and linked-basin options can be drawn from Fig. 8 where power generation average and variance is clus-

${ }_{425}$ tered relative to the $M_{2}$ cycle timing that it occurs. Ebb-only configurations feature on average the highest generation peak, followed by two-way configurations. There is a significant variance in power generated by single-basin systems attributed to spring-neap tide transitions. In contrast, the variance is significantly lower for linked-basin systems (as illustrated for LB- $N_{t}=4$, LB- $N_{t}=10$ 430 and LB- $\beta=25$ ). Nevertheless, Fig. $8 \mathrm{p}$ clearly demonstrates that the power generated is substantially lower than for single-basin tidal power plants.

\subsubsection{Thetis operation modelling results}

Thetis 2-D results are reported to affirm the 0-D trends observed for the operation of alternative lagoon design options remain valid once the effects of

435 regional hydrodynamics are included (Table 4). This exercise ensures 0-D model simplifications do not compromise predictive capability, by neglecting hydrodynamic impacts [18, 19]. In all cases considered, energy output 0-D/2-D model deviations are sufficiently small to support conclusions from both approaches. The deviations are indicated in Table 4 for completeness, and are largely within 440 the range of $\pm 10 \%$. The level of agreement indicates that both tidal range energy concepts function as expected even when acknowledging hydrodynamics impacts caused by the presence of the impoundment and the operation of the hydraulic structures. 

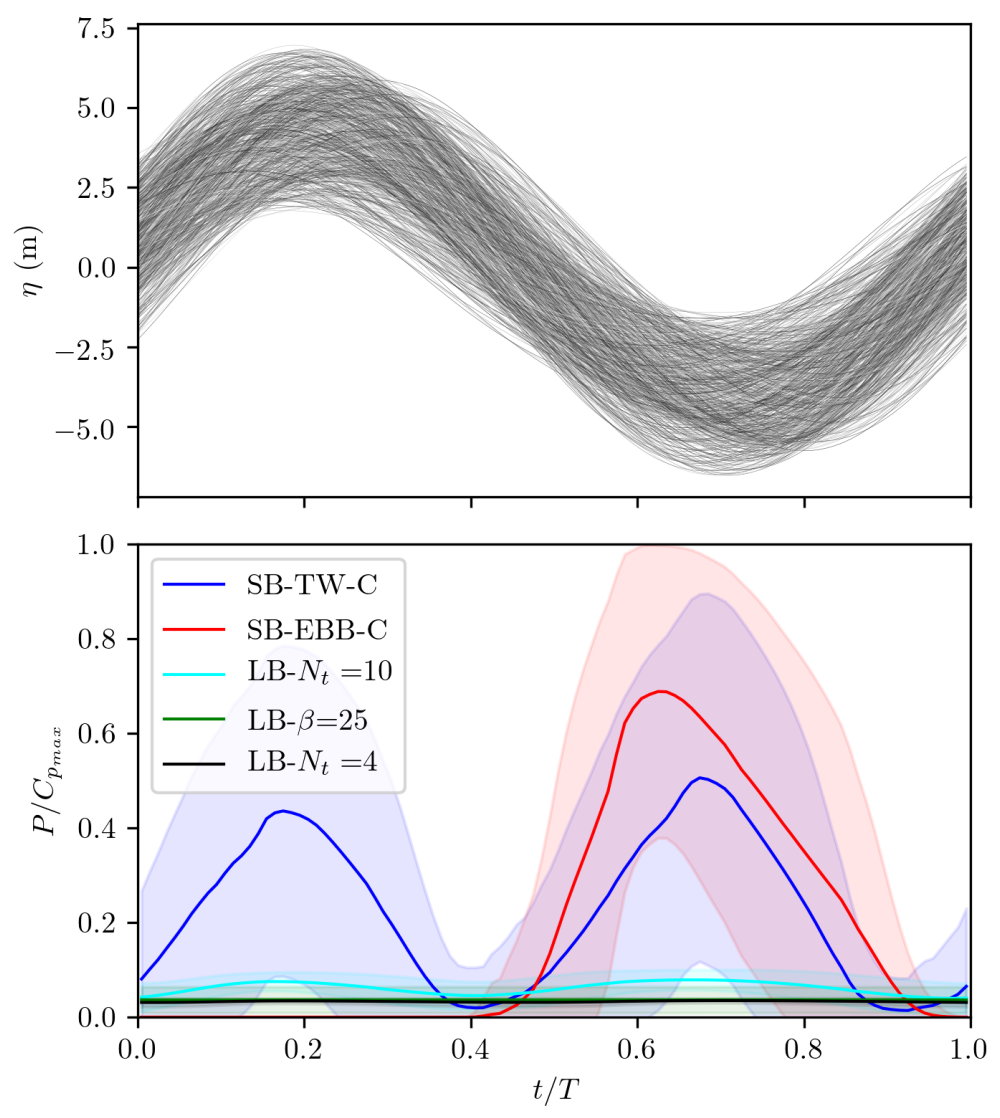

Figure 8: (a) Water elevations $(\eta)$ for each of the $12.42 \mathrm{~h}$ tidal cycles over a yearly period at the seaward side of the southern hydraulic structure section (top figure) and (b) average power-production over the tidal cycle period from annual 0-D simulations. Shaded areas illustrate the standard deviation of power produced at any point over the tidal cycle. $C_{p_{\max }}$ corresponds to the maximum capacity installed, i.e. $1960 \mathrm{MW}$ for single-basin configurations.

\section{Discussion}

Turbine design has a significant impact on tidal power plant performance. The hydraulic efficiency $\left(\eta_{h}\right.$, Table 1) of low-head turbines improves with $H$, explaining the reduced efficiency whilst $\alpha \leq 3.0 \mathrm{~m}$. Counter-intuitively, the efficiency for $\alpha>4.5 \mathrm{~m}$ is shown (Fig. 6p) to plateau and then decline. For high values of $\alpha$, efficiency is seen to decline for small values of $\lambda$ due to insufficient space to install the required capacity to exploit the resource. These effects are 
Table 4: Comparison between $0-\mathrm{D}$ and 2-D predictions for conventional and twin-basin systems considered. $E_{m t h, 0 D}=$ Monthly 0-D simulation prediction, $E_{m t h, 2 D}=$ Monthly 2-D simulation prediction

\begin{tabular}{l|ccc}
\hline Configuration & $\begin{array}{c}E_{m t h, 0 D} \\
(\mathrm{GWh})\end{array}$ & $\begin{array}{c}E_{m t h, 2 D} \\
(\mathrm{GWh})\end{array}$ & $\begin{array}{c}\frac{E_{m t h, 0 D}-E_{m t h, 2 D}}{E_{m t h, 0 D}} \\
(\%)\end{array}$ \\
\hline SB-EBB-C & 258.2 & 241.3 & 6.5 \\
SB-EBB-A & 260.2 & 233.3 & 10.3 \\
SB-TW-C & 319.4 & 308.2 & 3.5 \\
SB-TW-A & 367.8 & 349.4 & 5.0 \\
LB- $N_{t}=4$ & 46.6 & 48.3 & -3.6 \\
LB- $N_{t}=10$ & 85.3 & 93.3 & -9.4 \\
LB- $N_{t}=25$ & 108.9 & 97.3 & 10.6 \\
LB- $\beta=5.0$ & 76.9 & 75.6 & 1.6 \\
LB- $\beta=10.0$ & 58.6 & 58.9 & -0.5 \\
LB- $\beta=25.0$ & 51.5 & 50.8 & 1.4 \\
\hline
\end{tabular}

compounded by the inability of turbines to generate beyond their rated capacity. This applies when $H>H_{r}$; in our scenarios $H_{r}=5.0$, based on the turbine characteristics used previously [14. While turbines operate at peak capacity $\left(C_{p}\right)$, as $H>H_{r}$, a fraction of the flow expected through a conventional orifice equation is curtailed since only the flow required to generate $P_{h}=C_{p}$ is allowed through the caissons (Table 1. \#5-6). In the absence of sluice gates to further support the water exchange, this flow curtailment compromises generation for following cycles. However, a tidal range of $>12 \mathrm{~m}$ (represented in our sinusoidal signal by $\alpha>6 \mathrm{~m}$ ), is present at limited sites and only over spring tide condi460 tions. Examples include the extraordinary settings of the Severn Estuary, UK and the Bay of Fundy, Canada [4] where the tide exceeds a 12-14 $\mathrm{m}$ range during spring tides without any contribution from extreme weather events [20].

As formulations presented in Table 1 define the maximum energy and flow through the turbines as a function of $H$, a consistent power output is only facilitated once $H \geq H_{r}$. As such, $N_{t}$ for the linked-basin configuration with $\beta$ $=25.0$ is increased once $\alpha>3 \mathrm{~m}$ (see Fig. 6c) as only then can $H_{H W, L W} \geq$ $H_{r}=5 \mathrm{~m}$ be sustained. Tuning the turbine operation would be an option for $H_{H W, L W}<H_{r}$, but generation efficiency would further drop, allowing a greater discharge through the caissons relative to the power generated [13. Installation of superior and more dynamic turbines could improve efficiency, at the expense of further construction and O\&M costs. Nevertheless, idealised parameterisations (that exclude efficiency constraints over different conditions) were not considered as we sought to highlight the technology limitations at the time of writing.

475 The linked-basin system was initially optimised on the basis of maximising energy output as per the setup LB- $N_{t}=24$. The power generation profile in Fig. 7 suggests limited benefits in operating a linked-basin system to maximise energy. Specifically, the power generation profile of $\mathrm{LB}-N_{t}=24$ remains variable with distinct no-generation periods (Fig. 7b) whilst the energy output is a small 

linked-basin system to generate consistently (case LB- $\beta=25.0$ ) at no point does the number of turbines exceed $N_{t}=14$. The performance of the adaptive strategy in terms of energy output is $<10 \%$ of the overall potential energy and $<50 \%$ of what the system would generate if the objective were to maximise energy

${ }_{485}\left(\mathrm{LB}-N_{t}=24\right)$. More substantially, in comparison to the best performing singlebasin case of SB-TW-A, the linked-basin system delivers only $14 \%$ (or $14.5 \%$ according to 2-D results, Table 4) of the energy for the same area. On the other hand, the operation of LB- $\beta=25.0$ succeeds in providing continuous power albeit the gradual transitions in the power capacity used between tidal cycles.

490 The smoother transition in the power generation would potentially correspond to more manageable slew rates from an electronics point of view.

Comparisons between 0-D and 2-D in Section 3.2.3 reinforce the use of 0-D modelling for the energy output assessment of either single-basin or linked-basin schemes. Caution should however be exercised on this point when transferring the methodology to other sites. The use of $0-\mathrm{D}$ modelling is fitting at early design stages or for the conceptual development and comparison of tidal range energy options. For practical applications 0-D/2-D model results are aligned while 0-D model assumptions (listed in Prandle [8] ) and parameterisations adequately represent the system. This typically applies for relatively small lagoons

500 (e.g the Swansea Bay lagoon proposal of $A=11.6 \mathrm{~km}^{2}$ [45]) and/or schemes that do not interfere significantly with the seaward hydrodynamics.

Hydrodynamic models are essential to address hydro-environmental impacts and visualise how tidal range power plants would function in practise. Fig. 3 illustrates an instantaneous velocity contour plot from Thetis, indicating the general flow direction in the linked-basin system and also the general arrangement of the linked-basin system. As the shape and size of the linked-basin system closely follows the single-basin proposal of TLP's Cardiff Lagoon, a similar hydrodynamic impact is felt on the seaward tidal constituents as with the previous modelling results of Angeloudis and Falconer [17]. Upon construction, 510 the principal tidal constituents $M_{2} \approx 4.17 \mathrm{~m}$ and $S_{2} \approx 1.47 \mathrm{~m}$ would be reduced by $0.25 \mathrm{~m}$ and $0.12 \mathrm{~m}$ respectively, slightly affecting the pronounced tidal range in the Severn Estuary. Moreover, as the scheme acts as an obstruction to the otherwise unhindered basin, advective accelerations are noticeable as the flow is redirected to circumvent the impounded area. Inside the lagoons the 515 average velocity magnitude reduces away from the hydraulic structures; however, since turbines are consistently operating and promoting mixing, water within the linked-basin system would appear to be less prone to the effects of stagnation. These aspects should be more rigorously quantified by linking the hydrodynamics with water quality and morphodynamics models.

520 In terms of the energy outputs for both single-basin and linked-basin designs, further performance improvements could be realised if pumping periods are included. This entails the investment of energy to utilise turbines as pumping mechanisms at certain periods of the tidal cycle. The additional pumping function can lead to increases in the overall energy outputs of a scheme [46, 47. ${ }_{525}$ However, as pumping is not featured in the particular linked-basin system con- 
figuration, it has been intentionally omitted from the analysis for simplicity. Nonetheless, a subset of linked-basin designs by Bernshtein [23] include the distribution of pumps across the impoundment to provide an additional, pumped storage function to the operation. For single-basin designs, operation featuring pumping intervals has already been applied in practice at the La Rance barrage in France. In addition, recent studies suggest that pumping could add substantial value to prospective tidal power plants, as it can offer additional flexibility for both maximising the energy outputs as well as the income from the plant operation [15].

${ }_{535}$ Finally, while this study explored performance differences among linkedbasin and single basin systems, we did not consider capital expenses incurred in the construction phases of the project. It is observed that linked-basin systems will require less turbines, but more sluice gates. Due to the additional internal barrier, embankment costs will generally be higher than for single-basin systems, unless there are mitigating geographic features that could be exploited during construction [26]. Overall, given the equivalent scale of infrastructure required and the lower energy output of linked-basin schemes, this would place the cost of energy notably higher than for conventional tidal power plants. Nonetheless, more elaborate economic analyses should be performed to assess the feasibility

545 on a case by case basis, particularly if the connected basins are seen as multi-use coastal reservoirs with benefits beyond power generation.

\section{Conclusions}

This study is the first to present operational modelling results investigating the potential of linked-basin tidal range power systems relative to conventional, ${ }_{550}$ single-basin tidal range power plants. A particular incentive of linked-basin options is the potential to deliver continuous, sustainable power to the electricity grid. However, we highlight that the varying tidal range can greatly influence the magnitude of the power that can be consistently delivered.

Depth-averaged regional hydrodynamics simulations demonstrate that a twinbasin system could extract at best $\approx 30 \%$ of the energy of a conventional tidal power plant occupying an equivalent plan surface area. If power generation consistency is prioritised, the power output drastically reduces to a significantly smaller fraction; for a practical case considered for a prominent tidal range energy site, the linked-basin system delivers only $14 \%$ in comparison with a stan560 dard single-basin two-way generation configuration of equivalent size. Nevertheless, it is suggested that linked-basin designs may feature hydro-environmental advantages that include the continuous exchange of water volumes across connected basins (avoiding stagnation) and the deployment of a reduced number of turbines that generate over a greater Capacity Factor. In addition, turbines in the linked-basin system are confined in the interior barrier suggesting that their operation is unlikely to interfere with marine migratory routes. The latter is because only sluice gate hydraulic structures would be positioned on the seaward side of the impoundment. 
Finally, simulations show that linked-basin concepts are technically feasible. However, the power generation consistency that linked-basin systems can offer comes at the expense of a significantly lower extractable energy that can be exploited. Considering the low generation efficiency of linked-basin schemes, their value remains questionable taking into account recent advances in novel energy storage options.

\section{Acknowledgements}

A. Angeloudis acknowledges the support of the NERC Industrial Innovation fellowship grant NE/R013209/2. M. Piggott acknowledges the support of EPSRC under grants EP/M011054/1 and EP/R029423/1. We would also like to express our gratitude towards the anonymous reviewers for their insightful and 580 comprehensive comments that helped improve the manuscript prior to publication.

\section{References}

[1] A. G. Borthwick, Marine renewable energy seascape, Engineering 2 (2016) $69-78$.

[2] M. Melikoglu, Current status and future of ocean energy sources: A global review, Ocean Engineering 148 (2018) 563 - 573.

[3] R. Martin-Short, J. Hill, S. Kramer, A. Avdis, P. Allison, M. Piggott, Tidal resource extraction in the pentland firth, uk: Potential impacts on flow regime and sediment transport in the inner sound of stroma, Renewable Energy 76 (2015) $596-607$.

[4] Y. H. Bae, K. O. Kim, B. H. Choi, Lake Sihwa tidal power plant project, Ocean Engineering 37 (2010) 454-463.

[5] S. Waters, G. Aggidis, Tidal range technologies and state of the art in review, Renewable and Sustainable Energy Reviews 59 (2016) 514-529.

[6] M. Kadiri, R. Ahmadian, B. Bockelmann-Evans, W. Rauen, R. Falconer, A review of the potential water quality impacts of tidal renewable energy systems, Renewable and Sustainable Energy Reviews 16 (2012) 329-341.

[7] C. Hendry, The role of tidal lagoons, Technical Report, UK Government, 2017. URL: https://hendryreview.com/.

[8] D. Prandle, Simple theory for designing tidal power schemes, Advances in water resources 7 (1984) 21-27.

[9] G. Aggidis, D. Benzon, Operational optimisation of a tidal barrage across the Mersey estuary using 0-D modelling, Ocean Engineering 66 (2013) 69-81. 
[10] R. A. Falconer, A. Angeloudis, R. Ahmadian, Modeling Hydroenvironmental Impacts of Tidal Range Renewable Energy Projects in Coastal Waters, WORLD SCIENTIFIC, 2018, pp. 1553-1574. doi 10. 1142/9789813204027_0055.

[11] J. Wolf, I. A. Walkington, J. Holt, R. Burrows, Environmental impacts of tidal power schemes, Proceedings of the ICE Maritime Engineering 162 (2009) 165-177.

[12] M. Lewis, A. Angeloudis, P. Robins, P. Evans, S. Neill, Influence of storm surge on tidal range energy, Energy 122 (2017) 25 - 36.

[13] G. Aggidis, O. Feather, Tidal range turbines and generation on the solway firth, Ren. Energy 43 (2012) 9 - 17.

[14] A. Angeloudis, S. C. Kramer, A. Avdis, M. D. Piggott, Optimising tidal range power plant operation, Applied Energy 212 (2018) 680 - 690.

[15] F. Harcourt, A. Angeloudis, M. D. Piggott, Utilising the flexible generation potential of tidal range power plants to optimise economic value, Applied Energy 237 (2019) $873-884$.

[16] N. Čož, R. Ahmadian, R. A. Falconer, Implementation of a full momentum conservative approach in modelling flow through tidal structures, Water 11 (2019).

[17] A. Angeloudis, R. A. Falconer, Sensitivity of tidal lagoon and barrage hydrodynamic impacts and energy outputs to operational characteristics, Ren. Energy 114(A) (2017) 337-351.

[18] N. Yates, I. Walkington, R. Burrows, J. Wolf, The energy gains realisable through pumping for tidal range energy schemes, Renewable Energy 58 (2013) 79-84.

[19] R. Burrows, I. Walkington, N. Yates, T. Hedges, J. Wolf, J. Holt, The tidal range energy potential of the West Coast of the United Kingdom, Applied Ocean Research 31 (2009) 229-238.

[20] S. P. Neill, A. Angeloudis, P. E. Robins, I. Walkington, S. L. Ward, I. Masters, M. J. Lewis, M. Piano, A. Avdis, M. D. Piggott, G. Aggidis, P. Evans, T. A. Adcock, A. Židonis, R. Ahmadian, R. Falconer, Tidal range energy resource and optimization - past perspectives and future challenges, Renewable Energy (2018) -.

[21] B. F. de Belidor, Architecture hydraulique ou l'art de conduire, d'élever et de ménager les eaux pour les différents besoins de la vie, volume 2, 1739.

[22] L. B. Bernshtein, Tidal power development-a realistic, justifiable and topical problem of today, IEEE Transactions on Energy Conversion 10 (1995) 591-599. 
[23] L. B. Bernshtein, Tidal energy for electric power plants, Israel Program for Scientific Translations, Jerusalem, Israel, 1961.

645

650

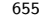

[24] Z. J. Wang, Z. W. Wang, A review on tidal power utilization and operation optimization, IOP Conference Series: Earth and Environmental Science 240 (2019) 052015.

[25] Y. Li, D.-Z. Pan, The ebb and flow of tidal barrage development in zhejiang province, china, Renewable and Sustainable Energy Reviews 80 (2017) 380 -389 .

[26] W. Van Walsum, Offshore Engineering For Tidal Power, in: The Ninth International Offshore and Polar Engineering Conference, International Society of Offshore and Polar Engineers, Brest, France, 1999, pp. 777-784.

[27] W. Van Walsum, Barriers to tidal power: multi basin plants, 65 2004. URL: http://www.waterpowermagazine.com/features/ featurebarriers-to-tidal-power-multi-basin-plants/.

[28] A. Baker, Tidal power, IEE Proceedings A Physical Science, Measurement and Instrumentation, Management and Education, Reviews 134 (1987) 392.

[29] J. Zhou, S. Pan, R. a. Falconer, Optimization modelling of the impacts of a Severn Barrage for a two-way generation scheme using a Continental Shelf model, Ren. Energy 72 (2014) 415-427.

[30] C. Zhu, R. H. Byrd, P. Lu, J. Nocedal, Algorithm 778: L-bfgs-b: Fortran subroutines for large-scale bound-constrained optimization, ACM Trans. Math. Softw. 23 (1997) 550-560.

[31] Poyry, Levelised cost of power from tidal lagoons, A report to Tidal Lagoon Power Plc, 2014. Accessed: 15-10-2017.

[32] R. Burrows, I. Walkington, N. Yates, T. Hedges, J. Wolf, J. Holt, Tapping the Tidal Potential of the Eastern Irish Sea, Final Report, Joule Project JIRP106/03. University of Liverpool and Proudman Oceanographic Laboratory., 2009. URL: http://www.liv.ac.uk/engineering/tidalpower.

[33] F. Rathgeber, D. A. Ham, L. Mitchell, M. Lange, F. Luporini, A. T. T. Mcrae, G.-T. Bercea, G. R. Markall, P. H. J. Kelly, Firedrake: Automating the finite element method by composing abstractions, ACM Trans. Math. Softw. 43 (2016) 24:1-24:27.

[34] T. Kärnä, S. C. Kramer, L. Mitchell, D. A. Ham, M. D. Piggott, A. M. Baptista, Thetis coastal ocean model: discontinuous galerkin discretization for the three-dimensional hydrostatic equations, Geoscientific Model Development Discussions 2018 (2018) 1-36. 
[35] T. Karna, B. de Brye, O. Gourgue, J. Lambrechts, R. Comblen, V. Legat, E. Deleersnijder, A fully implicit wetting-drying method for DG-FEM shallow water models, with an application to the Scheldt Estuary, Computer Methods in Applied Mechanics and Engineering 200 (2011) 509-524.

[36] S. Balay, S. Abhyankar, M. F. Adams, J. Brown, P. Brune, K. Buschelman, L. Dalcin, V. Eijkhout, W. D. Gropp, D. Kaushik, M. G. Knepley, L. C. McInnes, K. Rupp, B. F. Smith, S. Zampini, H. Zhang, H. Zhang, PETSc Users Manual, Technical Report ANL-95/11 - Revision 3.7, Argonne National Laboratory, 2016. URL: http://www.mcs.anl.gov/petsc

[37] A. Angeloudis, R. Falconer, S. Bray, R. Ahmadian, Representation and operation of tidal energy impoundments in a coastal hydrodynamic model, Renewable Energy 99 (2016) 1103-1115.

[38] A. Angeloudis, R. Ahmadian, R. A. Falconer, B. Bockelmann-Evans, Numerical model simulations for optimisation of tidal lagoon schemes, Applied Energy 165 (2016) 522-536.

[39] B. B. Parker, Tidal analysis and prediction., NOAA, NOS Center for Operational Oceanographic Products and Services (2007).

[40] TLP, Tidal Lagoon Power Cardiff Lagoon, http://www. tidallagoonpower.com/projects/cardiff/planning/, 2017. Accessed: 15-10-2017.

[41] A. Avdis, A. S. Candy, J. Hill, S. C. Kramer, M. D. Piggott, Efficient unstructured mesh generation for marine renewable energy applications, Renewable Energy 116 (2018) 842-856.

[42] G. D. Egbert, S. Y. Erofeeva, Efficient inverse modeling of barotropic ocean tides, Journal of Atmospheric and Oceanic Technology 19 (2002) 183-204.

[43] Edina Digimap Service, Hydrospatial one, gridded bathymetry, http:// digimap.edina.ac.uk/marine/, 2014. , SeaZone Solutions Ltd, Online; accessed 2017.

[44] A. Cornett, J. Cousineau, I. Nistor, Assessment of hydrodynamic impacts from tidal power lagoons in the Bay of Fundy, International Journal of Marine Energy 1 (2013) 33-54.

[45] S. Waters, G. Aggidis, A world first: Swansea bay tidal lagoon in review, Renewable and Sustainable Energy Reviews 56 (2016) 916 - 921.

[46] N. Yates, I. Walkington, R. Burrows, J. Wolf, Appraising the extractable tidal energy resource of the UK's western coastal waters, Phi. Trans. Royal Soc. A: Math. Phys. Eng. Sci. 371 (2013).

[47] A. Angeloudis, Tidal range structure operation assessment and optimisation, Dams and Reservoirs 0 (0) 1-10. 